\title{
Fisioterapia motora na prevenção e tratamento da osteopenia da prematuridade: uma revisão integrativa
}

\author{
Physiotherapy in the prevention and treatment of prematurity osteopenia: an integrative review
}

\author{
Sílvia Raquel Jandta, Laís Rodrigues Gerzson ${ }^{b}$, Carla Skilhan de Almeidac \\ a Fisioterapeuta. Pós-graduada em Fisioterapia em Pediatria e Neonatologia Hospitalar pela FISIOWORK - Faculdade Redentor, Rio de Janeiro, RJ, Brasil. \\ Unidade de Neonatologia do Hospital de Clínicas de Porto Alegre, RS, Brasil. \\ b Fisioterapeuta. Doutoranda em Saúde da Criança e do Adolescente pela Universidade Federal do Rio Grande do Sul (UFRGS), Porto Alegre, RS, Brasil. \\ c Fisioterapeuta. Doutora em Ciência do Movimento Humano pela UFRGS. Docente do Curso de Fisioterapia da UFRGS, Porto Alegre, RS, Brasil.
}

RESUMO

Introdução: A cada ano, um em cada 10 bebês, em todo o mundo, nasce prematuro os quais são suscetíveis a osteopenia da prematuridade. A osteopenia da prematuridade é caracterizada por alterações de mineralização esquelética decorrentes do deficiente acréscimo do conteúdo mineral ósseo.

Objetivo: Revisar na literatura atual os ensaios clínicos randomizados sobre os efeitos da fisioterapia motora nos recém-nascidos prematuros em relação à prevenção e/ou tratamento da osteopenia da prematuridade, bem como, observar quais as técnicas mais utilizadas nessa assistência.

Materiais e Métodos: Trata-se de uma revisão integrativa da literatura, incluindo ensaios clínicos randomizados que utilizaram técnicas de fisioterapia motora como intervenção. Buscou-se nas principais bases de dados (Pubmed, Cochrane Central, Lilacs e PEDro), estudos publicados de 2005 até 2016, utilizando os descritores: "prematuro", "mineralização óssea", "fisioterapia", "osteopenia da prematuridade" e seus equivalentes em inglês.

Resultados: Foram incluídos sete estudos e as técnicas utilizadas foram mobilizações passivas nas articulações dos membros superiores e inferiores, com suaves compressões articulares e um protocolo de estimulação tátil-cinestésica, massagem atividade física previne o desequilíbrio entre a formação e absorção da matriz óssea.

Conclusão: Embora haja poucos ensaios clínicos randomizados, pode-se observar que a fisioterapia motora utiliza técnicas que são efetivas no ganho de peso, crescimento, massa muscular e na prevenção do desequilíbrio entre a formação e absorção da matriz óssea em prematuros, tendo um papel importante na prevenção e no tratamento da osteopenia da prematuridade.

Palavras-chave: prematuro; fisioterapia; doenças ósseas metabólicas.

ABSTRACT
Introduction: Each year, one in 10 babies are born premature worldwide and are susceptible to osteopenia of prematurity. Osteopenia of prematurity is characterized by alterations in skeletal mineralization due to deficient increase in bone mineral content.
Objective: To review, in the current literature, randomized clinical trials on the effects of motor physical therapy in preterm infants in relation to the prevention and/or treatment of prematurity osteopenia, as well as to observe the most used techniques for this assistance.
Materials and Methods: This is an integrative review of the literature including randomized clinical trials using motor physiotherapy as intervention techniques. We have searched the main databases (Pubmed, Cochrane Central, Lilacs and PEDro) for studies published from 2005 to 2016, using the descriptors: "premature", "bone mineralization", "physiotherapy", "osteopenia of prematurity" and its equivalents in Portuguese.
Results: Seven studies were included and the techniques used were passive mobilizations in the joints of the upper and lower limbs, with soft articular compression and a protocol of tactile-kinesthetic stimulation.
Conclusion: Although there are few randomized clinical trials, it was observed that motor physical therapy uses techniques that are effective in weight gain, growth, muscle mass and prevention of the imbalance between the formation and absorption of the bone matrix in premature infants, having an important role in the prevention and treatment of prematurity osteopenia.
Keywords: preterm; physiotherapy; metabolic bone diseases.

\section{Correspondência:}




\section{INTRODUÇÃO}

A matriz de proteína orgânica chamada osteóide e uma fase mineral composta principalmente de cálcio e cristais de hidroxiapatita fosforoso são os principais componentes estruturais do osso $^{1}$. A osteopenia da prematuridade (OP), também chamada de doença óssea metabólica da prematuridade ou raquitismo da prematuridade, pode ser definida como uma redução na matriz proteica orgânica (osteopenia) e ou uma redução do componente mineral (osteomalacia) com ou sem alterações raquíticas ${ }^{1,2}$.

A OP tem uma etiologia multifatorial. Além da ingestão inadequada de nutrientes (cálcio, fósforo e vitamina D), a duração prolongada da nutrição parenteral (PN), imobilização e medicamentos como diuréticos e esteróides também contribuem para o seu desenvolvimento ${ }^{2}$.

Sua incidência está amplamente associada à idade gestacional e ao peso de nascimento. Ocorre com mais frequência nos recém-nascidos com idade gestacional menor que 32 semanas e peso inferior a $1000 \mathrm{~g}$ e entre os que desenvolvem complicações clínicas no período pós-natal ${ }^{3}$. Aparecendo logo após o nascimento, suas manifestações clínicas iniciam entre a $6^{\underline{a}}$ e $12^{\underline{a}}$ semanas de vida, podendo evoluir com redução do crescimento longitudinal, alargamento epifisário dos ossos longos, maior frequência de escolioses e deformidades no crânio e na sua forma mais grave, a ocorrência de fraturas espontâneas ${ }^{4-6}$.

Muitos fatores contribuem para o aumento do risco de OP, como: a perda de grande parte da aquisição de minerais como o cálcio, o fósforo e o magnésio, nível de vitamina $\mathrm{D}$, que acontece no último trimestre da gestação; a interrupção do transporte placentário de estrogênio e outros hormônios que promovem formação óssea7,8. Além disso, após o nascimento, é difícil atender às necessidades minerais do prematuro, mesmo diante da administração de dietas especiais com fórmulas fortificadas. O acréscimo mineral no meio extrauterino não se equipara ao esperado no período gestacional ${ }^{9}$.

Para a prevenção e tratamento da OP, é fundamental que uma equipe multidisciplinar atue na prática clínica para prevenir ou reduzir os déficits sobre a mineralização óssea em prematuros de risco. A fisioterapia motora, juntamente com os avanços nutricionais e melhorias nos cuidados pósnatais nas unidades de terapia intensiva podem minimizar o decréscimo mineral ósseo e auxiliar no processo de ganho de massa óssea dos recém-nascidos prematuros ${ }^{10}$. A aplicação de forças mecânicas estimula a formação e o crescimento ósseo e a inatividade, predispõe a absorção ${ }^{11}$. Estudos realizados com crianças e adultos sugerem que a realização de programas de exercícios regulares, promovem melhor mineralização óssea e tem sido utilizado como prevenção e parte do tratamento da osteopenia em diferentes fases da vida, por induzir os osteoblastos a aumentar sua atividade em resposta ao estímulo mecânico ${ }^{12-14}$.

Pesquisas em prematuros sugerem que estimulações passivas diárias são benéficas na prevenção e no tratamento das doenças ósseas metabólicas, além de favorecer o ganho de peso nessas crianças, combinadas com uma dieta adequada $^{15,16,17,18}$. Assim, o objetivo deste estudo foi revisar na literatura atual os ensaios clínicos randomizados sobre os efeitos da fisioterapia motora nos recém-nascidos prematuros em relação à prevenção e/ou tratamento da osteopenia da prematuridade, bem como, observar quais as técnicas mais utilizadas nessa assistência.

\section{MATERIAIS E MÉTODOS}

Como critérios de elegibilidade, foram incluídos ensaios clínicos randomizados realizados com recém-nascidos prematuros, que utilizaram como intervenção técnicas de fisioterapia motora, e foram considerados como desfechos: medidas antropométricas, achados radiológicos, exames bioquímicos sanguíneos e marcadores urinários.

Na seleção dos estudos, os títulos e resumos de todos os artigos identificados pela estratégia de busca foram avaliados. Todos os resumos que não forneciam informações suficientes sobre os critérios de inclusão e exclusão foram selecionados para avaliação do texto completo. Nessa segunda fase, foram avaliados os textos completos dos artigos e a seleção foi feita de acordo com os critérios de elegibilidade especificados anteriormente. Como estratégia, foi realizada busca nas bases de dados MEDLINE (via PubMed), Cochrane Central, Lilacs e PEDro, publicados de janeiro de 2005 até dezembro de 2015, além de busca manual em referências de estudos já publicados sobre o assunto. Selecionou-se para análise materiais de 2005 até o que a literatura atual apresenta, para restringir a busca nos últimos dez anos.

Os artigos foram selecionados utilizando os seguintes termos: "premature infants", "bone mineralization", "bone metabolic desease", "osteopenia of prematurity", "physiotherapy", "physical activity" e seus equivalentes em português "prematuro", "mineralização óssea", "fisioterapia", "osteopenia da prematuridade", "atividade física", associados a seus termos sinônimos e uma lista de termos sensíveis para a busca por ensaio clínico randomizado. Critérios de inclusão do estudo: (1) artigos apresentados com texto na íntegra; (2) escritos em português ou inglês; (3) publicados entre 2005 e 2015; (4) que abordassem a fisioterapia motora como prevenção e/ou tratamento da osteopenia da prematuridade. Não houve restrições quanto à amostra para maximizar os resultados da pesquisa. Foram excluídos do estudo 
(1) monografias; (2) artigos de revisão; (3) anais de eventos;

(4) dissertações e teses; (5) estudos fora do período do estudo e (6) estudo com animais.

A extração dos dados foi realizada, utilizando um formulário padronizado. Foram extraídas informações com relação às características metodológicas dos estudos, ano, tipo de estudo, população, intervenções, comparação e desfechos, demonstrados na Tabela 1.

\section{RESULTADOS \\ Descrição dos estudos}

A estratégia de busca identificou 78 artigos, dos quais 21 foram considerados relevantes e retomados para análise detalhada, destes, 14 foram excluídos: 3 por se tratarem de experimentos com animais, 9 por não utilizar técnicas fisioterapêuticas, 2 por serem artigos de revisão. 7 estudos

Tabela 1. Descrição dos estudos incluídos na revisão.

\begin{tabular}{|c|c|c|c|c|}
\hline Estudo & Amostra & Intervenção & Marcadores & Desfechos \\
\hline Litmanovitz et al. ${ }^{22}$ & $\begin{array}{l}8 \mathrm{Gl} \text {; } \\
8 \mathrm{GC} \text {, peso < } 1500 \mathrm{~g} ; \\
\text { IG média } 27 \text { sem. }\end{array}$ & $\begin{array}{l}\text { Mobilização passiva }+ \\
\text { compressões articulares } \\
\text { suaves; } \\
5 x / \text { articulação (total } 10 \mathrm{~min} \text { ), } \\
5 x / \text { semana por } 8 \text { sem.; } \\
\text { GC - segurado/acariciado } \\
\text { por } 10 \text { min }\end{array}$ & $\begin{array}{l}\text { QUS da porção média da } \\
\text { tíbia, 1x/semana por } 8 \text { sem.; } \\
\text { coletado sangue antes e após } \\
\text { a sessão; } \\
\text { níveis de FAE e ICTP }\end{array}$ & $\begin{array}{l}\text { Redução da SOS tibial no GC nas } 4 \text { primeiras } \\
\text { semanas, já no GI não apresentou mudança. } \\
\text { Aumento na FAE e redução da ICTP nos } 2 \text { grupos. }\end{array}$ \\
\hline Moyer-Mileur et al. ${ }^{23}$ & $\begin{array}{l}11 \mathrm{GC} ; \\
11 \mathrm{GM} ; \\
11 \mathrm{Gl} ; \\
\text { IG 26-28 sem., } \\
\text { peso } 800-1200 \mathrm{~g} ; \\
\text { IG 29-31 sem., } \\
\text { peso } 1201-1550 \mathrm{~g}\end{array}$ & $\begin{array}{l}5-10 \text { min/dia, até } 2 \mathrm{~kg} ; \\
\mathrm{Gl} \text { - terapeuta que realizava; } \\
\mathrm{GM} \text { - terapeuta ensinava o } \\
\text { protocolo às mães; } \\
\mathrm{GC}-5 \text { min carinho e } \\
\text { interatividade }\end{array}$ & $\begin{array}{l}\text { FAE, Pyd. ind. antrop, dieta; } \\
\text { DEXA (ulna e radio) } \\
\text { analisando AO, CMO e } \\
\text { DMO }\end{array}$ & $\begin{array}{l}\text { Peso, AO, CMO e DMO maiores nos GI e GM do } \\
\text { que GC. FAE se manteve constante no Gl e GM, } \\
\text { no GC houve redução. Pyd não diferiu entre os } \\
\text { grupos. Efetividade da administração do protocolo } \\
\text { de atividade física no crescimento e mineralização } \\
\text { ósseos. }\end{array}$ \\
\hline Vignochi et al. ${ }^{15}$ & $\begin{array}{l}15 \mathrm{Gl} ; \\
14 \mathrm{GC} \text {; } \\
\text { IG inferior a } 35 \text { sem. }\end{array}$ & $\begin{array}{l}\text { 10x/articulação (total } 15 \mathrm{~min} / \\
\text { sessão), } 5 x / \text { semana até } \\
\text { atingirem } 2 \mathrm{~kg} \text {; } \\
\text { média de } 25 \text { dias de } \\
\text { intervenção }\end{array}$ & $\begin{array}{l}\text { Ind. antrop., DEXA o CMO, } \\
\text { DMO, massa musc, gordura } \\
\text { corporal (início e fim) }\end{array}$ & $\begin{array}{l}\text { O GI - média de ganho de peso e massa muscular e } \\
\text { crescimento maior que o GC. Gl - ganho de CMO e } \\
\text { DMO e de AO no decorrer do estudo. GC - perdas } \\
\text { com mesma oferta nutricional. Fisioterapia motora } \\
\text { passiva contribuiu na prevenção da osteopenia } \\
\text { da PMT. }\end{array}$ \\
\hline Chen et al. ${ }^{3}$ & $\begin{array}{l}8 \mathrm{GC} ; \\
8 \mathrm{Gl} \text {, peso <1500 g; } \\
\text { IG média } 28 \text { sem. }\end{array}$ & $\begin{array}{l}\text { 10x/articulação (total } 10 \mathrm{~min} \text { ), } \\
5 x / \text { semana por } 4 \text { sem. }\end{array}$ & $\begin{array}{l}\text { QUS da tíbia a cada } 2 \text { sem: } \\
\text { ao nascer, } 2^{a}, 4{ }^{a}, 6^{\underline{a}} \text { e } \\
8^{\underline{a}} \text { semana de vida; } \\
\text { Ca, Mg, FAE, osteocalcina/ } \\
\text { osteoprotegerina }\end{array}$ & $\begin{array}{l}\text { Ca, Mg = normais no GC e Gl. FAE = aumento nos } \\
\text { dois grupos, sem diferença estatística. } \\
\text { Osteocalcina = mais alta no GC na } 4^{a} \text { semana, e na } \\
2^{\text {a }} \text { no Gl. Osteoprotegerina = estável no GC e GI. } \\
\text { Diminuição da redução da SOS no GI na } 6^{\underline{a}} \text { e } 8^{\underline{a}} \text { sem. } \\
\text { Realização precoce de exercícios assistidos pode } \\
\text { melhorar a força nos RNPT, redução do declínio } \\
\text { pós-natal da SOS nos ossos, prevenindo a OP. }\end{array}$ \\
\hline Tosun et al. ${ }^{16}$ & $\begin{array}{l}20 \mathrm{GC} \text {; } \\
20 \mathrm{Gl} \text {, peso entre } \\
800 \text { e } 1600 \mathrm{~g} \text {; } \\
\text { IG } 26 \text { e } 32 \text { sem., } \\
\text { iniciando } 3 \text { dias de } \\
\text { vida }\end{array}$ & $\begin{array}{l}\text { 5x/articulação, } \\
5 x / \text { semana por } 4 \text { sem.; } \\
\text { GC recebeu apenas os } \\
\text { cuidados de enfermagem } \\
\text { de rotina }\end{array}$ & $\begin{array}{l}\text { Ind. Antrop., circunferência } \\
\text { do braço, comprimento } \\
\text { da tíbia; } \\
\text { QUS da tíbia, antes e após } \\
\text { o estudo }\end{array}$ & $\begin{array}{l}\text { Aumento significativo na SOS da tíbia no Gl e } \\
\text { redução no GC. A circunferência do braço no Gl } \\
\text { aumentou, sem alterações significativas nos demais } \\
\text { índices antropométricos. Programa de atividade } \\
\text { física diária afeta positivamente a força óssea e a } \\
\text { circunferência do braço dos RNPT. }\end{array}$ \\
\hline Vignichi et al. ${ }^{11}$ & $\begin{array}{l}15 \mathrm{Gl} ; \\
15 \mathrm{GC} ; \\
\text { IG inferior ou igual a } \\
35 \text { sem. }\end{array}$ & $\begin{array}{l}\text { Gl: exercícios 10x/articulação } \\
\text { ( } 15 \text { min/sessão), 5x/sem. até } \\
\text { os RNs atingirem } 2000 \text { g; } \\
\text { média de } 24 \text { dias de } \\
\text { intervenção }\end{array}$ & $\begin{array}{l}\text { Medidos no início e fim } \\
\text { do estudo: FAE, sangue, Dpd } \\
\text { urina, Ind. Antrop. e dados } \\
\text { maternos }\end{array}$ & $\begin{array}{l}\text { Sem diferenças entre os grupos da oferta de minerais, } \\
\text { vit. D, calorias na dieta, uso de antibióticos e } \\
\text { corticoides. Ganho de peso, níveis de FAE foram } \\
\text { maiores Gl e redução no valor da Dpd. GC obteve } \\
\text { aumento desse marcador. Aumento da FAE e a } \\
\text { redução da Dpd após exercícios mostra que o } \\
\text { desequilíbrio entre a formação e a reabsorção óssea } \\
\text { parece ser atenuado com a intervenção. }\end{array}$ \\
\hline Haley et al. ${ }^{21}$ & $\begin{array}{l}20 \mathrm{Gl} ; \\
20 \mathrm{GC} ; \\
\text { IG entre } 28-32 \text { sem. }\end{array}$ & $\begin{array}{l}\text { Massagem corporal } 20 \text { min; } \\
2 x / \text { dia e mobilização passiva, } \\
20 \text { min, } 2 x / \text { dia, } 6 \text { dias na } \\
\text { semana por } 15 \text { dias }\end{array}$ & $\begin{array}{l}\text { QUS da tíbia; Marcadores } \\
\text { urinários: Pyd, Dpd e } \\
\text { osteocalcina (U-MidOC e } \\
\text { unOC) }\end{array}$ & $\begin{array}{l}\text { SOS da tíbia foi reduzida no GC enquanto que o } \\
\text { GI teve os valores dentro da linha de base, sendo } \\
\text { maiores que GC. As diferenças da Dpd e Pyd } \\
\text { foram semelhantes entre os grupos. Osteocalcinas } \\
\text { aumentaram no GI. A TKS melhora a resistência } \\
\text { óssea em RNPT pois reduz a perda da densidade do } \\
\text { osso. Biomarcadores do metabolismo ósseo sugerem } \\
\text { modificação na remodelação óssea }\end{array}$ \\
\hline
\end{tabular}

RNPT: Recém-nascidos pré-termo; PMT: prematuro; GI: grupo intervenção; GC: grupo controle; GM: grupo mães; sem.: semanas; flex e ext: flexão e extensão; x: repetições do exercício (vezes); MsSs e Msls: membros superiores e inferiores; min: minuto; IG: idade gestacional; FAE: fosfatase alcalina espessífica; Pyd: pyridinium crosslinks-Piridinolina; Dpd: deoxipiridinolina urinária; AO: área óssea; SOS: velocidade do som; QUS: ultra-som quantitativo; DEXA: Dual Energy X-Ray Absorptiometry; CMO; conteúdo mineral ósseo; DMO: densidade mineral óssea; TKS: estimulação tátil-cinestésica; U-MidOC: osteocalcin midfragment; unOC: undercarboxylated osteocalcin; ICTP: carboxi-terminal cross links telopeptlde of type 1 colagenase; Vit: vitamina; Ind.Antrop.: índices antropométricos; PC e Tx: perímetro cefálico e torácico; UTINeo: unidade de tratamento intensivo neonatal. 
preencheram os critérios de elegibilidade e foram incluídos nesta revisão, totalizando 204 pacientes participantes. A

Figura 1 mostra o fluxograma dos estudos incluídos.

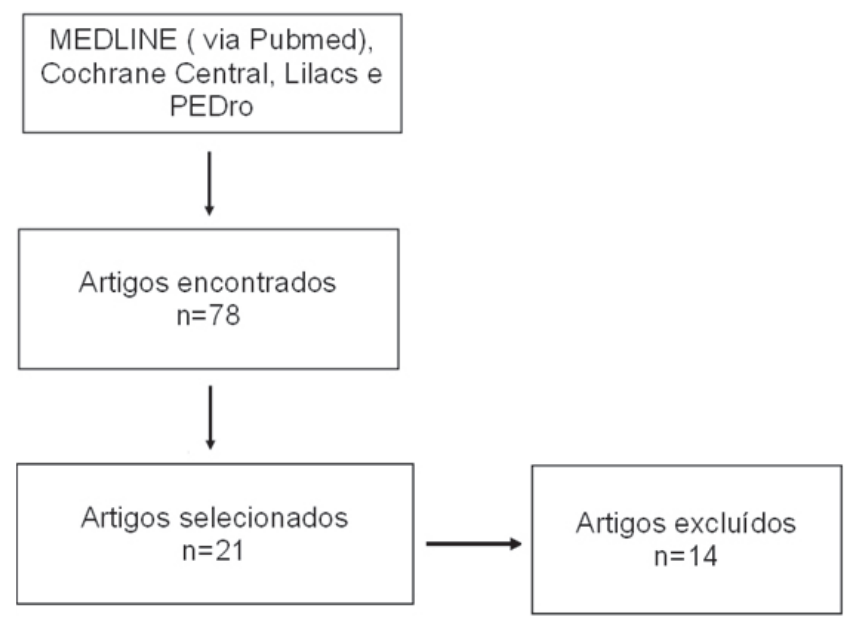

Figura 1. Fluxograma demonstrando a busca e seleção dos estudos.

As técnicas utilizadas como intervenção foram semelhantes entre os estudos, que se basearam em um protocolo que elaboraram um programa de mobilização passiva em flexão e extensão, com compressões articulares suaves nas articulações dos membros superiores (ombros, cotovelos e punhos) e inferiores (quadris, joelhos e tornozelos), diferindo apenas no número de sessões semanais e no número de repetições de cada mobilização ${ }^{19,20}$. Outro protocolo utilizou massagem além das mobilizações passivas. O tempo de intervenção variou de cinco a 20 minutos, numa frequência de cinco a seis dias por semana, pelo período de dois a oito semanas ${ }^{21}$.

\section{Efeitos das intervenções}

\section{Medidas antropométricas}

Todos os estudos determinaram, nos critérios de inclusão, valores nutricionais semelhantes na dieta dos pacientes, que deveriam estar recebendo alimentação enteral com média calórica $110 \mathrm{cal} / \mathrm{kg}$ por dia.

Os estudos variaram quanto à avaliação das medidas antropométricas dos RNPT (peso, comprimento, perímetro cefálico, abdominal e braquial, massa muscular). Três estudos, não analisaram tais medidas 3,21,22.

No estudo de Moyer-Mileur et al. ${ }^{23}$ havia dois grupos com 11 participantes cada, que receberam a mesma intervenção e um grupo controle. No primeiro grupo, o programa de mobilizações (GM) era realizado por um fisioterapeuta e, no segundo grupo, pelas mães dos pacientes (GMom) (treinadas seguindo o mesmo programa de mobilizações do fisioterapeuta). Observou-se, no final do estudo, um ganho maior de peso nos dois grupos que receberam a intervenção (GM: $844 \mathrm{~g}$, GMoml: $855 \mathrm{~g}$ ) se comparados ao grupo controle (GC: $725 \mathrm{~g} ; p=0,001$ ), já as demais medidas como comprimento, perímetro cefálico e braquial não diferiram significativamente entre os grupos. Vignochi et al. ${ }^{11,15}$ também relataram um ganho maior de peso por dia comparando grupo intervenção e controle $(27,43 \pm 3,73$ vs $21,01 \mathrm{~g} \pm 4,4$, $p<0,001)$ e de massa muscular por semana $(1,28 \pm 0,34 \mathrm{~cm}$ vs $0,78 \pm 0,23, p<0,001)^{14}$. No estudo de Tosun et al. ${ }^{16} \mathrm{a}$ circunferência do braço, que é um preditivo de crescimento ósseo, foi $35.71 \mathrm{~cm}$ para o grupo interventivo $(7,60 \pm 0,5)$ e $24.13 \mathrm{~cm}(7,2 \pm 0,5)$ para o Grupo Controle $(p<0,001)$. Os demais dados não diferiram de forma significativa.

\section{Alterações Radiológicas}

Alguns estudos utilizaram Ultrassom Quantitativo (QUS) da tíbia, com o intuito de avaliar a velocidade de propagação do som (SOS) antes e após a aplicação do programa de exercícios passivos ${ }^{3,16,21,22}$. Os achados dos três primeiros foram semelhantes, onde a SOS da tíbia apresentou valores reduzidos no GC e inalterados ou dentro da normalidade no Gl. Tosun et al. ${ }^{14}$ relataram aumento da SOS no Gl e redução no GC. Concluíram que o programa de exercícios passivos nos RNPT previne a redução da SOS dos ossos, que aconteceria normalmente pelo nascimento prematuro, melhorando a força e a resistência óssea nesta população.

Tanto Vignochi et al. ${ }^{11}$ como Moyer-Mileur et al. ${ }^{22}$ mediram a densitometria óssea por DEXA do antebraço (Dual energy X-Ray Absorptiometry) de corpo total. Analisaram o conteúdo mineral ósseo, a densidade mineral óssea e a área óssea. Ambos os estudos relataram um aumento nos valores das variáveis citadas após a realização da intervenção $(p<0,05)$, comparados aos grupos sem intervenção. Ressaltaram o impacto positivo da realização do programa de exercícios passivos sobre o crescimento ósseo e aquisição mineral dos RNPT, prevenindo a osteopenia no início da vida pós-natal ${ }^{15,22}$. Dados demonstrados nas Tabela 2 e $\mathbf{3}$ respectivamente.

Tabela 2. Alterações radiológicas referentes ao ultrassom quantitativo entre a primeira e a última análise da velocidade de propagação do som de cada estudo.

\begin{tabular}{lccc}
\hline \multicolumn{1}{c}{ Estudos } & $\begin{array}{c}\text { Grupo } \\
\text { Intervenção }\end{array}$ & Grupo Controle & $\boldsymbol{p}$ \\
\hline Chen et al. $^{3}$ & $-67,2 \pm 32,3^{*}$ & $-201,7 \pm 146,2^{*}$ & 0,020 \\
Haley et al. $^{20}$ & $\begin{array}{c}\text { constante } \\
\text { valores não } \\
\text { informados }\end{array}$ & $\begin{array}{c}\text { reduziu } \\
\text { valores não } \\
\text { informados }\end{array}$ & $<0,05$ \\
Litmanovitz et al. ${ }^{21}$ & $\begin{array}{c}11,3 \pm 22,8^{*} \\
\text { Tosun et al. }{ }^{15}\end{array}$ & $-108.1 \pm 33,7^{*}$ & $<0,0001$ \\
\hline
\end{tabular}

*Média e desvio padrão. **Mediana. 
Tabela 3. Diferença entre a primeira e a última mensuração da absorciometria por duplo raio-x.

\begin{tabular}{cccccc}
\hline Estudos & Variáveis & Grupo Controle & Grupo Interventivo & GMom & \multicolumn{1}{c}{$\boldsymbol{p}$} \\
Moyer-Mileur et al. ${ }^{22}$ & DMO & $0,005^{*}$ & $0,008^{*}$ & 0,010 & 0,12 \\
& CMO & $0,110^{*}$ & $0,278^{*}$ & 0,001 \\
Vignochi et al. ${ }^{10}$ & AO & $1,17^{*}$ & $2,72^{*}$ & -27 & 0,001 \\
& DMO & $-3,15^{*}$ & 8,37 & - & 0,001 \\
& CMO & $-8,18^{*}$ & 434,00 & - & 0,001 \\
\hline
\end{tabular}

GMom: Programa de mobilizações pelas mães dos pacientes. * Média.

\section{Exames Bioquímicos Sanguíneos}

Após o programa de exercícios, estudo relatou um aumento importante nos níveis da Fosfatase Alcalina Específica entre o grupo interventivo comparado com o grupo controle11,22,23. Apenas um estudo não encontrou diferença entre os grupos ${ }^{3}$. A Tabela 4 ilustra esses dados. Litmanovitz et al. ${ }^{21}$ analisou o biomarcador de reabsorção óssea ICTP (carboxi-terminal cross-links telopeptíde of type 1 colagen), e os resultados não foram esclarecedores, pois tanto o GC $(92,3 \pm 7,7)$ quanto o GI $(100,8 \pm 8,2)$ obtiveram redução significativa desses valores após a intervenção $(p<0,05)$. Concluíram que oito semanas de exercícios passivos previnem a redução da SOS óssea nessa população, se iniciado na primeira semana de vida. Isso sugere que a estimulação mecânica é benéfica para a força e metabolismo ósseo, e o exercício tem uma importante função no desenvolvimento ósseo no período pós-natal.

Tabela 4. Médias da Fosfatase alcalina específica, no início dos estudos e no final, após a intervenção.

\begin{tabular}{|c|c|c|c|c|c|}
\hline \multirow{2}{*}{ Estudos } & \multicolumn{2}{|c|}{ Grupo Interventivo } & \multicolumn{2}{|c|}{ Grupo Controle } & \multirow{2}{*}{$p$} \\
\hline & Pré & Pós & Pré & Pós & \\
\hline Litmanovitz et al..21 & 152,4 & 235,6 & 119,7 & 179,3 & 0,01 \\
\hline Moyer-Mileur et al. ${ }^{22}$ & - & 162,7 & - & 83,2 & 0,04 \\
\hline Chen et al. ${ }^{3}$ & 223,8 & 517,3 & 227,6 & 632,3 & 0,4 \\
\hline Vignochi et al. ${ }^{10}$ & 104,9 & 126,3 & 108,2 & 112,2 & 0,02 \\
\hline
\end{tabular}

Não foi realizada a correlação dos valores do ICTP entre os grupos, que parece ter deixado uma lacuna no resultado final da pesquisa.

\section{Exames Bioquímicos Urinários}

Moyer-Mileur et al. ${ }^{22}$ analisaram a excreção urinária da Piridinolina, um marcador de reabsorção óssea, verificaram que os valores se mantiveram próximos à linha de base em ambos os grupos. Tem se utilizado dele na prática clínica diária e é disponível com facilidade.

No estudo de Haley et al. ${ }^{20}$ foram analisados os marcadores de reabsorção óssea: Piridinolina, deoxipiridinolina e de formação óssea: osteocalcina, em duas formas: de médio fragmento e sob carboxilada. Os valores iniciais, ou de linha de base desses marcadores foram semelhantes nos dois grupos. Houve uma tendência de aumento da Piridinolina na segunda semana de intervenção $(p=0,08)$. As diferenças da Piridinolina e deoxipiridinolina entre o Gl e o GC foram similares, sem significância estatística $(p=0,9$ e $p=0,2)$. As osteocalcinas e a carboxilada aumentaram significativamente após duas semanas de intervenção no $\mathrm{Gl}(p<0,001)$, não sendo observado aumento no GC. Se comparados os valores entre os grupos, o Gl apresentou valores 32\% melhores da osteocalcinas que o controle. Ao final do estudo, referiram que o aumento das osteocalcinas sugerem remodelação óssea global nos prematuros que receberam a estimulação tátil-cinestésica, parecendo melhorar a força óssea dessa população.

Vignochi et al. ${ }^{11}$ analisaram a deoxipiridinolina somente. Encontraram aproximadamente $43 \%$ de redução no $\mathrm{Gl}$ até o final do estudo, enquanto que o GC apresentou aumento desse marcador $(p<0,001)$. Esses resultados demonstraram que o Gl apresentou uma desaceleração da reabsorção óssea no período pós-natal dos prematuros que receberam a fisioterapia motora. Conclui-se, assim que esses benefícios da prevenção e tratamento fisioterapêutico favorecem a qualidade óssea dos bebês prematuros.

\section{DISCUSSÃO}

Este estudo teve como objetivos revisar na literatura atual os ensaios clínicos randomizados a respeito dos efeitos da fisioterapia motora nos recém-nascidos prematuros sobre a prevenção e/ou tratamento da osteopenia da prematuridade, bem como, observar quais as técnicas mais utilizadas nessa assistência. As pesquisas demonstraram que programas de atividade física ou a fisioterapia motora não somente aumenta a mineralização e o crescimento ósseos como também previne a diminuição da resistência óssea, além de favorecer o ganho de peso dos recém-nascidos prematuros.

Pode-se identificar o aparecimento da osteopenia precocemente aos sinais clínicos e alterações radiológicas através das alterações em exames bioquímicos, sendo 
realizados rotineiramente através da mensuração sérica de cálcio total ou iônico, fósforo, magnésio e fosfatase alcalina. Outra maneira é a utilização de ultrassom quantitativo para avaliar a estrutura e a densidade óssea dos prematuros, e a coleta dos marcadores urinários como a Piridinolina e Deoxipiridinolina, entre outros, que refletem a reabsorção óssea (atividade osteoclástica) como métodos de avaliação não invasivo ${ }^{3,11}$. A absorciometria por duplo raio-x, que permite mensurar o conteúdo mineral e a densidade óssea, é a opção mais confiável para a realização de pesquisas, embora não seja utilizada clinicamente por ser pouco acessível. Já a radiografia comum é bastante utilizada na rotina hospitalar, embora exponha condições já avançadas de desmineralização óssea, pode ser útil na identificação de fraturas e deformidades esqueléticas ${ }^{5}$. Baixos níveis séricos de fósforo associados a altas taxas de reabsorção urinária do mineral, aumento da fosfatase alcalina sérica e hipercalciúria são indicativos de doença óssea metabólica ${ }^{5}$.

No presente artigo, pode-se evidenciar que a aplicação de um programa de exercícios passivos nas articulações dos membros superiores e inferiores, associando compressões articulares, bem como, a associação de protocolo de massagem, resultou em ganho de peso ${ }^{11,15,22}$, aumento da circunferência do braço $^{16}$, assim como do comprimento e da massa muscular dos pacientes ${ }^{15}$. Embora os estudos não relacionam esses achados com o tempo de internação, podese acreditar que essas crianças tenham menos complicações e recebam alta hospitalar mais rapidamente.

Observou-se também, através do Ultrassom Quantitativo, que a realização da intervenção evitou que a velocidade de propagação do som dos ossos diminuísse, ou seja, evitou a redução da resistência óssea, além de aumentá-la, demonstrando um maior efeito protetor sobre a perda óssea característica dos RNPT $3,16,21,22$. Clinicamente esses achados impactam positivamente na qualidade de vida destas crianças, proporcionando-as um desenvolvimento ósseo mais adequado, evitando fraturas e deformidades e a dor que elas causam.

Os resultados dos estudos da densitometria óssea revelaram aumento da densidade, do conteúdo mineral, bem como da área óssea, mostrando um impacto positivo na mineralização e no crescimento ósseo dessa população ${ }^{15,23}$. No estudo de Vignochi et al. ${ }^{11}$ foram relatados aumento significativo nos níveis do marcador de formação óssea e redução do marcador de reabsorção óssea deoxipiridinolina urinária. Já Haley et al..$^{21}$ relatou aumento nas osteocalcinas, marcadores urinários de formação óssea.

Os estudos demonstraram que os marcadores de formação e reabsorção óssea tenderam a aumentar ou a manter-se dentro da normalidade, sugerindo que a realização da fisioterapia pode prevenir o desequilíbrio entre formação e absorção de matriz óssea que normalmente ocorre nos RNPT devido a própria prematuridade. As alterações radiológicas parecem evidenciar o efeito positivo dos exercícios na população de prematuros, como demonstrado nos estudos já citados. Em concordância, Ocarino et al. ${ }^{24}$ pesquisaram o efeito da realização de atividade física sobre o desenvolvimento ósseo em ratas, evidenciando que aquelas que praticaram atividade física, além de apresentarem menor perda óssea, tiveram aumento da espessura cortical e do volume trabecular dos ossos. Esses resultados confirmam a evidência que o exercício pode prevenir ou tratar os efeitos da osteopenia.

Para Demarinii ${ }^{25}$, a osteopenia não deve ser subestimada pelos clínicos, apesar de ser considerada como doença de resolução espontânea após um período conhecido de desmineralização óssea durante os primeiros anos de vida. Isso porque ainda não se tem a clareza quanto à influência do desenvolvimento ósseo neonatal e dos primeiros anos de vida sobre o pico de massa óssea atingido na adolescência e idade adulta, o que determina o risco de desenvolvimento da osteoporose e fraturas futuramente ${ }^{26}$.

A aplicação rotineira de exercícios de mobilização passiva articular, massagem e posicionamento está relacionada ao ganho ponderal, ao aumento na densidade e no conteúdo mineral ósseo. Portanto, a implementação de fisioterapia motora parece proporcionar estabilidade ou estímulo para a formação óssea, dessa forma, prevenir e/ou minimizar as complicações decorrentes da doença óssea metabólica ${ }^{10}$.

Resultados semelhantes foram relatados por Aly et al. ${ }^{18}$ em pesquisa sobre os efeitos da massagem combinada a exercícios passivos em RNPT, evidenciando maior mineralização óssea no grupo da intervenção. Os autores explicaram que o processo de crescimento e metabolismo ósseo é complexo, envolvendo a deposição de matriz óssea com incorporação do conteúdo mineral que é equilibrada com reabsorção enzimática e remodelamento. Considerando que o exercício aumenta eficiência metabólica e os níveis de hormônios de crescimento em humanos, é plausível esperar que a atividade física, bem como a massagem, possa empurrar a balança a favor da formação óssea ${ }^{18}$.

O estudo acima citado, assim como outros ${ }^{3,22}$, relatam que a análise isolada dos marcadores de reabsorção óssea não parecem ser bons indicadores de doença óssea, devido a contínua reabsorção óssea e constante mobilização de cálcio no processo de mineralização.

Recentemente, Schulzke et al. ${ }^{28}$ publicaram uma revisão sistemática sobre atividade física para promover crescimento e mineralização óssea em bebês prematuros. Verificaram os efeitos dos exercícios passivos a curto e longo prazo. 
Relataram que a maioria das pesquisas apresenta resultados a curto prazo, somente uma mostrou benefícios 12 meses após o estudo. Concluíram que o programa de atividade física trouxe moderados benefícios a curto prazo nos participantes, dentre eles: ganho de peso, massa e área ósseas, maior crescimento dos participantes, porém a importância clínica desses achados ainda é questionável, devido a variabilidade dos estudos e pequeno número de participantes. Mais estudos que incorporam crianças com alto risco para a osteopenia devem ser realizados. Os ensaios clínicos neste estudo devem abordar eventos adversos, resultados a longo prazo, efeitos da ingestão nutricional (calorias, proteínas, cálcio, fósforo $)^{28}$.

Observou-se que houve uma semelhança em relação às técnicas de exercícios passivos realizados pelos estudos revisados, de maneira a sugerir a utilização, ainda que cautelosa, do protocolo de Moyer-Mileur et al. ${ }^{20}$ como uma intervenção da fisioterapia motora nas unidades de neonatologia.

Algumas limitações presentes no nosso estudo merecem ser destacadas. Primeiramente, os estudos incluídos na nossa revisão de literatura possuem diferentes métodos de aplicação da fisioterapia motora. Alguns utilizaram exclusivamente o protocolo de mobilizações passivas e compressões articulares; outros, associaram a ele um protocolo de massagem, isso dificulta a percepção de qual técnica é mais efetiva. O número de repetições e o tempo de tratamento variou bastante, bem como uma variância no tipo de marcador analisado por estudo. A amostra dos estudos foi relativamente pequena. Destaca-se que todos os estudos enfatizaram a importância da nutrição adequada em conjunto à aplicação de exercícios físicos nos prematuros. Além disso, a pesquisa analisou os artigos dos últimos 10 anos, o que de certa forma torna-se também uma limitação importante.

Através dessa revisão de literatura integrativa, pode-se observar que a fisioterapia motora, utiliza técnicas de mobilizações passivas das articulações dos membros superiores e inferiores e compressões articulares suaves, associadas ou não à massagem, é efetiva para o ganho de peso, crescimento, massa muscular, conforme os resultados das medidas antropométricas e exames bioquímicos, e do conteúdo e da densidade mineral óssea, verificados com a utilização da densitometria óssea e do ultrassom quantitativo, podendo auxiliar na prevenção e/ou tratamento da osteopenia. Mais estudos clínicos randomizados devem ser realizados para afirmar com segurança a aplicabilidade desse programa na rotina de cuidados em unidades neonatais, com um número maior de participantes, para dar aporte científico a essa modalidade de intervenção.

\section{REFERÊNCIAS}

1. Viswanathan S, Khasawneh W, McNelis K, Dykstra C, Amstadt R, Super DM, Groh-Wargo S, Kumar D. Metabolic bone disease: a continued challenge in extremely low birth weight infants. JPEN J Parenter Enteral Nutr. 2014;38(8):982-90. https://doi. org/10.1177/0148607113499590

2. Mannan MA, Jahan I, Rahman MZ, Hasan Z, Dey AC, Shahidullah M. Osteopenia of Prematurity: Are We at Risk? Mymensingh Med J. 2015;24(3):631-7.

3. Chen HL, Lee $\mathrm{CL}$, Tseng $\mathrm{H}$, Yang SN, Yang RC, Jao HC. Assisted exercise improves bone strength in very low birthweight infants by bone quantitative ultrasound. J Paediatr Child Health. 2010;46(11): 653-9. https://doi.org/10.1111/j.1440-1754.2010.01822.x

4. Yeşiltepe Mutlu G, Kırmızıbekmez H, Ozsu E, Er I, Hatun S.. Metabolic bone disease of prematurity: report of four cases. J Clin Res Pediatr Endocrinol. 2014;6(2):111-5. https://doi.org/10.4274/ jcrpe. 1282

5. Bozzetti V, Tagliabue P. Metabolic bone disease in preterm newborn: an update on nutritional issues. Ital J Pediatr. 2009;35(1):20. https:// doi.org/10.1186/1824-7288-35-20

6. Catache M, Leone CR. Análise crítica dos aspectos fisiopatológicos, diagnósticos e terapêuticos da doença metabólica óssea em recémnascidos de muito baixo peso. J Pediatr. 2001;77(supl1):S53-S62. https://doi.org/10.2223/JPED.219

7. Dokos C, Tsacalidis C, Tragiannidis A, Rallis D. Inside the "fragile" infant: pathophysiology, molecular background, risk factors and investigation of neonatal osteopenia. Clin Cases Miner Bone Metab. 2013; 10(2):86-90

8. Karras SN, Fakhoury H, Muscogiuri G, Grant WB, van den Ouweland JM, Colao AM, Kotsa K. Maternal vitamin D levels during pregnancy and neonatal health: evidence to date and clinical implications. Ther Adv Musculoskelet Dis. 2016;8(4): 124-35. https://doi.org/10.1177/1759720X16656810

9. Field T, Diego MA, Hernandez-Reif M, Deeds O, Figuereido B. Moderate versus light pressure massage therapy leads to greater weight gain in preterm infants. Infant Behav Dev. 2006;29(4):574-8. https://doi.org/10.1016/j.infbeh.2006.07.011

10. Moreno J, Fernandes LV, Guerra CC. Fisioterapia motora no tratamento do prematuro com doença metabólica óssea. Rev Paul Pediatr. 2011;29(1):117-21. https://doi.org/10.1590/S010305822011000100018

11. Vignochi CM, Silveira RC, Miura E, Canani LHS, Procianoy RS. Physical therapy reduces bone resorption and increases bone formation in preterm infants. Am J Perinatol. 2012;29(8):573-8. https://doi.org/10.1055/s-0032-1310520

12. Rautava E, Lehtonen-Veromaa $M$, Kautiainen $H$, Kajander $S$, Heinonen OJ, Viikari J, Möttönen T. The reduction of physical activity reflects on the bone mass among young females: a follow-up study of 142 adolescent girls. Osteoporos Int. 2007;18(7):915-22. https://doi.org/10.1007/s00198-006-0312-x

13. Bayramoglu M, Sozay S, Karatas M, Kilink S. Relationships between muscle strength and bone mineral density of three body regions in sedentary postmenopausal women. Osteoporos Int. 2007;18(7):915-22. 
14. Pitukcheewanont P, Punyasavatsut N, Feuille M. Physical activity and bone health in children and adolescents. Pediatr Endocrinol Rev. 2010;7(3):275-82.

15. Vignochi CM, Miura E, Canani LH. Effects of motor physical therapy on bone mineralization in premature infants: a randomized controlled study. J Perinatol. 2008;28(9):624-31. https://doi.org/ 10.1038/jp.2008.60

16. Tosun O, Bayat M, Günes T, Erden E. Daily physical activity in low-risk pre-terms infants: positive impact on bone strength and mid-upper arm circum ference. Ann Hum Biol. 2011;38(5):635-9. https://doi.org/10.3109/03014460.2011.598187

17. Schulzke SM, Kaempfen S, Trachsel D, Patole SK. Physical activity programs for promoting bone mineralization and growth in preterm infants. Cochrane Database Syst Rev. 2014;(4):CD005387. https:// doi.org/10.1002/14651858.CD005387.pub3

18. Aly H, Moustafa MF, Hassanein SM, Massaro AN, Amer HA, Patel K. Physical activity combined with massage improves bone mineralization in premature infants: a randomized trial. J Perinatol. 2004;24(5):305-9. https://doi.org/10.1038/sj.jp.7211083

19. Moyer-Mileur LJ, Luetkemeier M, Boomer L, Chan GM. Effect of physical activity on bone mineralization in premature infants. J Pediatr. 1995;127(4):620-5. https://doi.org/10.1016/S00223476(95)70127-3

20. Moyer-Mileur LJ, Brunstetter V, McNaught TP, Gill G, Chan GM. Daily physical activity program increases bone mineralization and growth in preterm very low birth weight infants. Pediatrics. 2000;106(5):1088-92. https://doi.org/10.1542/peds.106.5.1088

21. Haley S, Beachy J, Ivaska KK, Slater H, Smith S, Moyer-Mileur LJ. Tactile/kinesthetic stimulation (TKS) increases tibial speed of sound and urinary osteocalcin (U-MidOC and unOC) in premature infants (29-32 wks PMA). Bone. 2012;51(4):661-6 https://doi. org/10.1016/j.bone.2012.07.016

22. Litmanovitz I, Dolfin T, Arnon S, Regev RH, Nemet D, Eliakim A. Assisted exercise and bone strength in preterm infants. Calcif Tissue Int. 2007;80(1):39-43. https://doi.org/10.1007/s00223-0060149-5

23. Moyer-Mileur LJ, Ball SD, Brunstetter VL, Chan GM. Maternaladministered physical activity enhances bone mineral acquisition in premature very low birth weight infants. J Perinatol. 2008;28(6): 432-7. https://doi.org/10.1038/jp.2008.17

24. Ocarino NM, Marubayashi U, Cardoso TG, Guimaraes CV, Silva AE, Torres RC, Serakides R. Physical activity in osteopenia treatment improved the mass of bones directly and indirectly submitted to mechanical impact. J Musculoskelet Neuronal Interact. 2007;7(1):84-93.

25. Demarini S. Calcium and phosphorus nutrition in preterm infants. Acta Paediatr Suppl. 2005;94(449):87-92. https://doi.org/ 10.1080/08035320510043619

26. Fewtrell, M. Early nutritional predictors of long-term bone health in preterm infants. Curr Opin Clin Nutr Metab Care. 2011;14(3): 297-301. https://doi.org/10.1097/MCO.0b013e328345361b

27. Hovi $P$, Andersson $S$, Järvenpää $A L$, Eriksson JG, Strang-Karlsson $S$, Kajantie E, Mäkitie $O$. Decreased bone mineral density in adults born with very low birth weight: a cohort study. PLoS Med. 2009;6(8):e1000135. https://doi.org/10.1371/journal. pmed.1000135

28. Schulzke SM, Kaempfen S, Trachsel D, Patole SK. Physical activity programs for promoting bone mineralization and growth in preterm infants. Cochrane Database Syst Rev. 2014;(4):CD005387. https:// doi.org/10.1002/14651858.CD005387.pub3 